\title{
Establishment of a 5-fluorouracil-resistant triple-negative breast cancer cell line
}

\author{
KATSUYUKI TAKAHASHI $^{1,2}$, MASAKO TANAKA ${ }^{1}$, AZUSA INAGAKI $^{3}$, \\ HIDEKI WANIBUCHI ${ }^{3}$, YASUKATSU IZUMI ${ }^{1}$, KATSUYUKI MIURA $^{4}$, \\ KATSUYA NAGAYAMA ${ }^{2}$, MASAYUKI SHIOTA ${ }^{1}$ and HIROSHI IWAO ${ }^{1}$ \\ ${ }^{1}$ Department of Pharmacology, Osaka City University Medical School; ${ }^{2}$ Department of Pharmacy, \\ Osaka City University Hospital; ${ }^{3}$ Department of Pathology, ${ }^{4}$ Applied Pharmacology \\ and Therapeutics, Osaka City University Medical School, Osaka, Japan
}

Received July 24, 2013; Accepted September 11, 2013

DOI: $10.3892 /$ ijo.2013.2135

\begin{abstract}
Triple-negative breast cancers (TNBCs) are defined as tumors that lack expression of estrogen receptor, progesterone receptor and human epidermal growth factor receptor 2. Clinically, TNBC patients are treated with cytotoxic drugs including 5-fluorouracil (5-FU). However, TNBCs develop resistance to such drugs after a series of treatments. To elucidate the mechanisms of drug resistance, establishment of drug-resistant cancer cell lines should be one of the most useful model systems. However, 5-FU-resistant TNBC cell lines have not been previously reported. In this study, we established a 5-FU-resistant cell line, MDA-MB-231/5-FU, from the human TNBC cell line MDA-MB-231, by repeated exposure to stepwise increases in the concentration of 5-FU. The $\mathrm{IC}_{50}$ value of 5-FU for MDA-MB-231/5-FU was 5.5-fold that for the parental cells. The MDA-MB-231/5-FU cell line acquired resistance to not only 5-FU, but also vinorelbine, paclitaxel and gemcitabine. Additionally, we performed iTRAQ-based quantitative proteomics in MDA-MB231/5-FU cells and the parental cells in order to characterize MDA-MB-231/5-FU. The proteins upregulated in the newly established cells were mainly classified into the categories of 'DNA recombination', 'cell cycle', 'complex assembly', 'cytoskeleton organization', 'transport' and 'negative regulation of cell death'. These proteins may be related to mechanisms of drug resistance in TNBCs. Our established MDA-MB231/5-FU cell line should be a useful tool for identifying new mechanisms of drug resistance and new drug targets in TNBCs.
\end{abstract}

Correspondence to: Dr Masayuki Shiota, Department of Pharmacology, Osaka City University Medical School, 1-4-3 Asahimachi, Abeno-ku, Osaka 545-8585, Japan

E-mail: sio@med.osaka-cu.ac.jp

Key words: drug-resistant cell, triple-negative breast cancer, 5-fluorouracil

\section{Introduction}

Triple-negative breast cancers (TNBCs) are defined as tumors that lack expression of estrogen receptor (ER), progesterone receptor (PR), and human epidermal growth factor receptor 2 (HER2) (1). TNBC patients account for 11-23\% of all breast cancers (2-4). TNBCs follow a more aggressive clinical course than other forms, such as luminal A and luminal B, and have a poor prognosis (4). They also have no indications for hormonal therapy or anti-HER 2 therapy. Therefore, treatment of TNBC patients is restricted to cytotoxic drugs such as 5-fluorouracil (5-FU), vinorelbine (VNB), paclitaxel (PTX), doxorubicin (DOX), and gemcitabine (GEM) (5). However, TNBCs acquire resistance to cytotoxic drugs after a series of treatments (6). The development of resistance to cytotoxic drugs appears to have become a major clinical problem in the chemotherapy of TNBCs.

Drug efflux mechanisms are the most well-studied mechanisms of drug resistance. The $\mathrm{ABC}$ family proteins, which include multidrug resistance protein 1 (MDR1) and breast cancer resistance protein (BCRP), are target molecules to overcome drug resistance (6-8). However, the combination of these protein inhibitors and cytotoxic drugs failed to show an improved outcome over cytotoxic drugs alone $(9,10)$.

Fluoropyrimidine anticancer drugs, as represented by 5-FU and capecitabine, have been used to treat various cancers and accepted worldwide as first-line anticancer drugs for breast cancers (11). The mechanisms of resistance to 5-FU, namely, enhanced activities of thymidylate synthase (TS) and dihydropyrimidine dehydrogenase (DPYD), are well known to endow cancer cells with resistance to 5 -FU in vitro and in clinical studies (12-15). Actually, 5-FU is used in combination with 5-chloro-2,4-dihydroxypyridine, which is a DPYD inhibitor. However, the effects of this combination are insufficient. The elucidation of other mechanisms of resistance to 5-FU are thus anticipated. Investigations of other mechanisms of 5-FU resistance may lead to the development of novel effective anticancer chemotherapies for 5-FU-resistant patients. To elucidate mechanisms of drug resistance, the establishment of drug-resistant cancer cell lines should be one of the most useful approaches for developing model systems (16-18). 
However, 5-FU-resistant TNBC cell lines have not been previously reported, although there have been some 5-FU-resistant lines of other forms of breast cancer or other tumors (11).

In this study, we established a 5-FU-resistant cell line, MDA-MB-231/5-FU, from the human TNBC cancer cell line MDA-MB-231, by repeated exposure of cells to stepwise increases in the concentration of 5-FU. Then, we applied a proteomic approach and the quantification of protein expression to compare proteins between MDA-MB-231/5-FU and MDA-MB-231 and identify those with differential expression. MDA-MB-231/5-FU may be a useful tool for identifying new mechanisms of drug resistance and new drug targets in TNBCs.

\section{Materials and methods}

Chemicals and antibodies. 5-FU, DOX and VNB were purchased from Kyowa Hakko (Tokyo, Japan), CDDP from Pfizer (New York, NY, USA), PTX from Bristol-Myers (New York, NY, USA), and GEM from Eli Lilly (Indianapolis, IN, USA). MDR1, p53 and phospho-p53 (Ser15) were purchased from Cell Signaling Technology (Beverly, MA, USA), DPYD and TS from GeneTex (San Antonio, TX, USA), BCRP from Abcam (Cambridge, UK), and $\beta$-actin from Sigma (St. Louis, MO, USA). Horseradish peroxidase (HRP)-conjugated secondary antibodies were purchased from GE Healthcare (Little Chalfont, Bucks, UK).

Cell lines and culture conditions. The human breast carcinoma cell line MDA-MB-231 was purchased from the American Type Culture Collection (ATCC, Rockville, MD, USA). Cells were cultured in Dulbecco's modified Eagle's medium (DMEM; Wako, Osaka, Japan) with 10\% fetal bovine serum (FBS) (Equitech-Bio, Kerrville, TX, USA), $100 \mathrm{U} / \mathrm{ml}$ penicillin, and $100 \mu \mathrm{g} / \mathrm{ml}$ streptomycin (Gibco, Grand Island, NE, USA) at $37^{\circ} \mathrm{C}$ under $5 \% \mathrm{CO}_{2}$ and $20 \% \mathrm{O}_{2}$ in a humidified chamber.

Establishment of 5-FU-resistant cell line. 5-FU-resistant cells were established from MDA-MB-231 by exposure to increasing concentrations of 5-FU. MDA-MB-231 were exposed to an initial 5-FU concentration of $3.84 \mu \mathrm{mol} / 1$ in DMEM plus $10 \%$ FBS. The drug concentration was then increased 1.25 times at each step of resistance, from $3.84 \mu \mathrm{mol} / 1$ up to $23.0 \mu \mathrm{mol} / 1$. Cells were cultured for at least four weeks at each step, with medium exchange every three days. Chemotherapeutic drugs were eliminated from the 5-FU-resistant MDA-MB-231 (MDA-MB-231/5-FU) for 15 days before each experiment.

Cell proliferation assays. Cell proliferation was examined using a Cell Counting Kit-8 (Dojindo Laboratories, Kumamoto, Japan) in accordance with the manufacturer's instructions. Briefly, a suspension of MDA-MB-231 or MDA-MB-231/5-FU (1.5x10 cells/well) in $100 \mu 1$ of DMEM with $10 \%$ FBS was seeded to 96 -well plates, and supplemented with 5-FU, DOX, CDDP, VNB, PTX and GEM. After incubation for $72 \mathrm{~h}$, Cell Counting Kit- 8 reagent was added to each well. After incubation for $90 \mathrm{~min}$, the cell viability was measured as absorbance at $450 \mathrm{~nm}$ using a microplate reader (Perkin-Elmer, Waltham, MA, USA). Analyses of all samples were performed in triplicate. The percentage of cell viability was determined as the ratio of absorbance of the sample versus that without $5-\mathrm{FU}$ as a control. The $\mathrm{IC}_{50}$ of a chemotherapeutic drug was determined as the concentration at which 50\% inhibition of cell growth was shown compared with the control cell growth.

Protein extraction. Cells were washed with PBS and lysed in lysis buffer consisting of $50 \mathrm{mM}$ HEPES, $\mathrm{pH} 8.0,150 \mathrm{mM}$ $\mathrm{NaCl}, 5 \mathrm{mM}$ EDTA, $1 \% \mathrm{NP}-40,10 \%$ glycerol, $100 \mathrm{mM} \mathrm{NaF}$, $1 \mathrm{mM}$ phenylmethylsulfonyl fluoride and protease inhibitor cocktail (Nacalai Tesque Inc., Kyoto, Japan). Lysates were separated by centrifugation, the supernatant was recovered, and protein concentrations were assayed using the bicinchoninic acid protein assay reagent (Thermo Fisher Scientific, Rockford, IL, USA).

iTRAQ sample labeling. TheiTRAQ analysis was performed in a double duplex manner. Protein lysates $(170 \mu \mathrm{g})$ from MDA-MB-231 and MDA-MB-231/5-FU were digested with trypsin and labeled with 114 and 117 iTRAQ reagents according to standard procedures.

Protein identification and relative quantification. Proteomic analysis was performed on a DiNa-AI Nano LC System (KYA Technologies, Tokyo, Japan) coupled to a QSTAR Elite hybrid mass spectrometer (AB Sciex, Framingham, MA, USA) through a NanoSpray ion source (AB Sciex) as previously described (19). Briefly, mobile phase A was $98 \%$ water [2\% acetonitrile (ACN), $0.1 \%$ formic acid], and mobile phase B was $70 \% \mathrm{ACN}$ ( $0.1 \%$ formic acid, $30 \%$ water). The column effluent was introduced into the spray chamber through a tapered stainless steel emitter and directly electrosprayed into the QSTAR System ion trap mass spectrometer in the positive mode for nano-electrospray ionization-MS/MS analysis. Each sample was run for $150 \mathrm{~min}$. Protein identification was performed using Analyst QS Software 2.0 (AB Sciex) in the positive-ion mode. Both sets of data were processed using ProteinPilot Software 2.0.1 with the Paragon ${ }^{\mathrm{TM}}$ search algorithm (AB Sciex). MS/MS data were searched against the NCBI database (RefSeq release 54 of July 2012 from the website ftp://ftp.hgc. jp/pub/mirror/ncbi/refseq/) using a Homosapiens taxonomy filter. The minimum threshold for protein identification was set at a protein score of 0.47 , corresponding to a confidence level $>66 \%$ and $1 \%$ false discovery rate.

Annotation analysis. GI accession numbers were uploaded into the DAVID 6.7 (Database for Annotation, Visualization, and Integrated Discovery) information tool. For Gene Ontology (GO) term analysis, we studied the 'Biological Process' categories using the GO FAT default settings. For functional annotation searches, we set the following parameters: 'Biological Process', threshold count 3, EASE 0.5; for functional annotation clusters, medium stringency. Enrichment values (GO terms), enrichment scores (annotation clusters), and statistical determinants (Fisher's Exact P-values) are those calculated using DAVID 6.7 software.

Western blotting. The lysates for western blotting (20 $\mu \mathrm{g}$ of protein) were separated on sodium dodecyl sulfatepolyacrylamide gels under reducing conditions, followed 
Table I. Cross-resistance of MDA-MB-231/5-FU cells.

\begin{tabular}{|c|c|c|c|c|c|c|c|c|c|c|c|c|}
\hline \multirow[b]{2}{*}{ Cell line } & \multicolumn{2}{|c|}{$5-\mathrm{FU}$} & \multicolumn{2}{|c|}{ DOX } & \multicolumn{2}{|c|}{ CDDP } & \multicolumn{2}{|c|}{ VNB } & \multicolumn{2}{|c|}{ PTX } & \multicolumn{2}{|c|}{ GEM } \\
\hline & $\begin{array}{c}\mathrm{IC}_{50} \\
(\mu \mathrm{mol} / \mathrm{l})\end{array}$ & RI & $\begin{array}{c}\mathrm{IC}_{50} \\
(\mathrm{nmol} / \mathrm{l})\end{array}$ & RI & $\begin{array}{c}\mathrm{IC}_{50} \\
(\mu \mathrm{mol} / \mathrm{l})\end{array}$ & RI & $\begin{array}{c}\mathrm{IC}_{50} \\
(\mathrm{nmol} / \mathrm{l})\end{array}$ & RI & $\begin{array}{c}\mathrm{IC}_{50} \\
(\mathrm{nmol} / \mathrm{l})\end{array}$ & RI & $\begin{array}{c}\mathrm{IC}_{50} \\
(\mathrm{pmol} / \mathrm{l})\end{array}$ & RI \\
\hline MDA-MB-231 & 29.9 & $5.5^{\mathrm{a}}$ & 38.2 & 1.3 & 2.0 & 0.7 & 2.1 & $2.5^{\mathrm{b}}$ & 1.1 & $8.4^{\mathrm{a}}$ & 33.4 & $8.1^{\mathrm{a}}$ \\
\hline MDA-MB-231/5-FU & 165.5 & & 49.3 & & 1.4 & & 5.2 & & 9.5 & & 270.1 & \\
\hline
\end{tabular}

The resistance index (RI) was determined as the ratio of $\mathrm{IC}_{50}$ between MDA-MB-231/5-FU and MDA-MB-231. The IC ${ }_{50}$ of MDA-MB231/5-FU to 5-FU, vinorelbine (VNB), paclitaxel (PTX) and gemcitabine (GEM) were significantly increased compared with those of parent

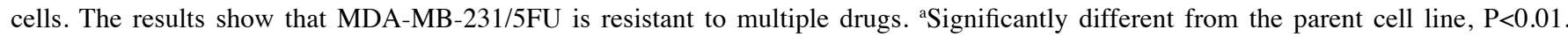
${ }^{\mathrm{b}}$ Significantly different from the parent cell line, $\mathrm{P}<0.05$.

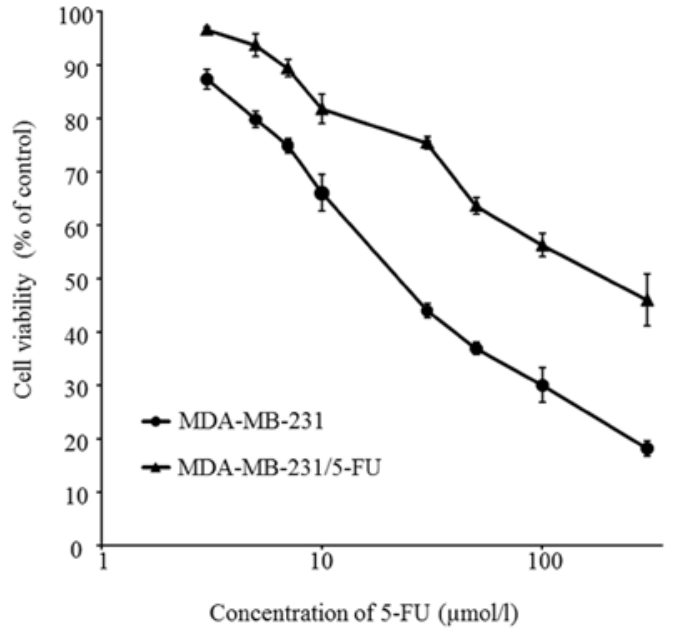

Figure 1. Dose response curve of MDA-MB-231 and MDA-MB-231/5-FU to 5-FU. MTS assay was carried out after $72 \mathrm{~h}$ of exposure to 5-FU. Dose response curve plotted from the results of MTS assays performed four times. Both cell lines displayed dose-dependent inhibition of cell growth. The $\mathrm{IC}_{50}$ values to 5-FU for MDA-MB-231 and MDA-MB-231/5-FU were 29.9 \pm 2.3 and $165.5 \pm 21.8 \mu \mathrm{M}$, respectively $(\mathrm{P}<0.01)$. The MDA-MB-231/5-FU cells were thus more resistant to 5 -FU. The results are expressed as the means \pm standard error.

by electrophoretic transfer to polyvinylidene difluoride membranes (Immobilon-P; Millipore, Billerica, MA, USA). After blocking, the membranes were probed with the appropriate primary antibodies. Membrane-bound primary antibodies were detected using secondary antibodies conjugated with HRP. The chemiluminescence was detected with LAS-4000 (GE Healthcare) using the enhanced chemiluminescence technique and quantified using Image Quant TL software (GE Healthcare).

\section{Results}

Establishment of 5-FU-resistant TNBC cell line. To explore the mechanisms of resistance to 5-FU, we established a 5-FU-resistant TNBC cell line. To achieve this, a human TNBC cell line, MDA-MB-231, was treated continuously with stepwise increases of the concentration of 5-FU every four weeks from $3.84 \mu \mathrm{mol} / 1$ to $23.0 \mu \mathrm{mol} / 1$. Fig. 1 shows cell survival curves of MDA-MB-231 and 5-FU-resistant cells. The cells were treated with different concentrations of 5-FU for $72 \mathrm{~h}$. The $\mathrm{IC}_{50}$ values of parent cells and 5-FU-resistant cells to $5-\mathrm{FU}$ were $29.9 \pm 2.3$ and $165.5 \pm 21.8 \mu \mathrm{mol} / \mathrm{l}$, respectively $(\mathrm{P}<0.01)$. The new cells were thus successfully established as a 5-FU-resistant TNBC cell line: MDA-MB-231/5-FU.

Cross-resistance profiles of MDA-MB-231/5-FU cells. MDA-MB-231/5-FU acquired resistance to 5-FU; its resistant index (RI) was 5.5. Generally, multiple drug resistance involves resistance to one drug accompanied by resistance to several other anticancer drugs (16). Therefore, we evaluated whether MDA-MB-231/5-FU acquired cross-resistance to other anticancer drugs used for TNBCs or with other mechanisms of action. The $\mathrm{IC}_{50}$ and $\mathrm{RI}$ are summarized in Table I. The $\mathrm{IC}_{50}$ values of parent cells to DOX, CDDP, VNB, PTX and GEM were $38.2 \pm 3.3 \mathrm{nmol} / 1,2.0 \pm 0.3 \mu \mathrm{mol} / 1,2.1 \pm 0.8 \mathrm{nmol} / 1$, $1.1 \pm 0.7 \mathrm{nmol} / 1$ and $33.4 \pm 5.7 \mathrm{pmol} / 1$, respectively. In contrast, the $\mathrm{IC}_{50}$ values of MDA-MB-231/5-FUto DOX, CDDP, VNB, PTX and GEM were $49.3 \pm 1.8 \mathrm{nmol} / 1,1.4 \pm 0.2 \mu \mathrm{mol} / \mathrm{l}$, $5.2 \pm 0.9 \mathrm{nmol} / 1,9.5 \pm 2.0 \mathrm{nmol} / 1$ and $270.1 \pm 15.4 \mathrm{pmol} / 1$, respectively. The RI of DOX, CDDP, VNB, PTX and GEM were $1.3,0.7,2.5,8.4$ and 8.1, respectively. MDA-MB-231/5-FU acquired cross-resistance to VNB, PTX and GEM. However, these cells were sensitive to DOX and CDDP.

Western blot analysis of proteins related to drug resistance. According to previous studies, the mechanisms of resistance to 5-FU involve increases in 5-FU-degrading enzyme DPYD and 5-FU-targeting enzyme TS (11-15,20). On the other hand, $\mathrm{ABC}$ family proteins, such as MDR1 and BCRP, are related to multiple drug resistance in breast cancer (6-8). To confirm the expression of proteins related to drug resistance, we examined MDR1, BCRP, DPYD and TS expression by western blot analysis. MDA-MB-231/5-FU showed increased levels of MDR1 and BCRP1 proteins compared with the parent cells (Fig. 2A). In contrast, there were no significant differences in DPYD and TS between the parent cells and MDA-MB-231/5-FU.

p53 plays a major role in cellular responses to DNA damage and other genomic aberrations (21). Activation of p53 can lead to cell cycle arrest, DNA repair, or apoptosis. Generally, phosphorylation of p53 is increased by DNA damage due to 5-FU (22-25). To evaluate the response to DNA damage, MDA-MB-231 and MDA-MB-231/5-FU were treated with $30 \mu \mathrm{M} 5$-FU for 6,12 and $24 \mathrm{~h}$. The phosphorylation 
A

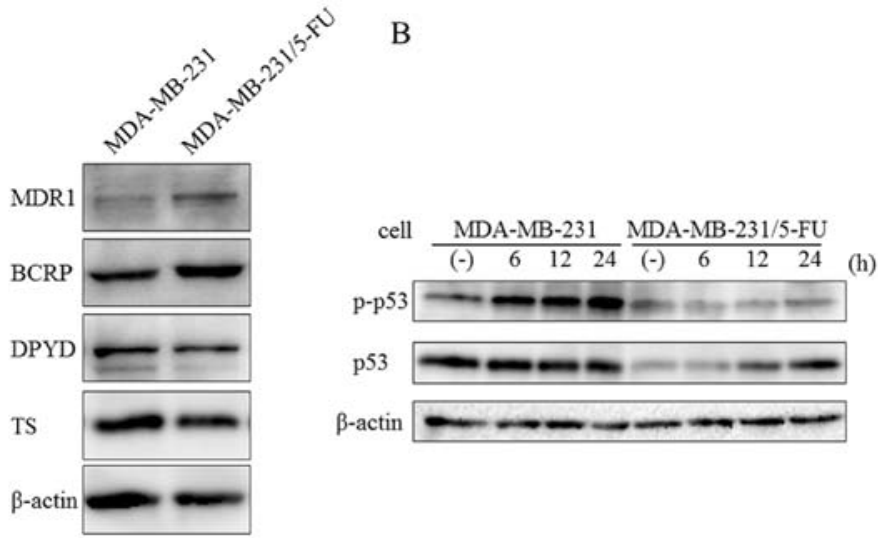

Figure 2. Western blot analysis of proteins related to drug resistance. After cells were harvested at $80 \%$ confluence, total proteins were extracted by the protocol described in Materials and methods. (A) MDA-MB-231/5-FU cells exhibited increased levels of MDR1 and BCRP proteins compared with MDAMB-231. $\beta$-actin was used as a loading control. (B) MDA-MB-231 and MDA-MB-231/5-FU were treated with $30 \mu \mathrm{M} 5$-FU (approximately the IC s0 $_{50}$ of parent cells). MDA-MB-231 cells exhibited increased levels of p53 and phospho-p53 proteins.

\begin{tabular}{|c|c|c|c|c|c|c|c|c|}
\hline $\begin{array}{c}\text { DNA } \\
\text { recombination }\end{array}$ & cell cycle & $\begin{array}{c}\text { complex } \\
\text { assembly }\end{array}$ & $\begin{array}{c}\text { cytoskeleton } \\
\text { organization }\end{array}$ & Transport & $\begin{array}{c}\text { negative regulation } \\
\text { of cell death }\end{array}$ & $\begin{array}{c}\text { chromatin } \\
\text { organization }\end{array}$ & $\begin{array}{c}\text { cell } \\
\text { differentiation }\end{array}$ & \begin{tabular}{c} 
Other \\
\hline
\end{tabular} \\
\hline
\end{tabular}
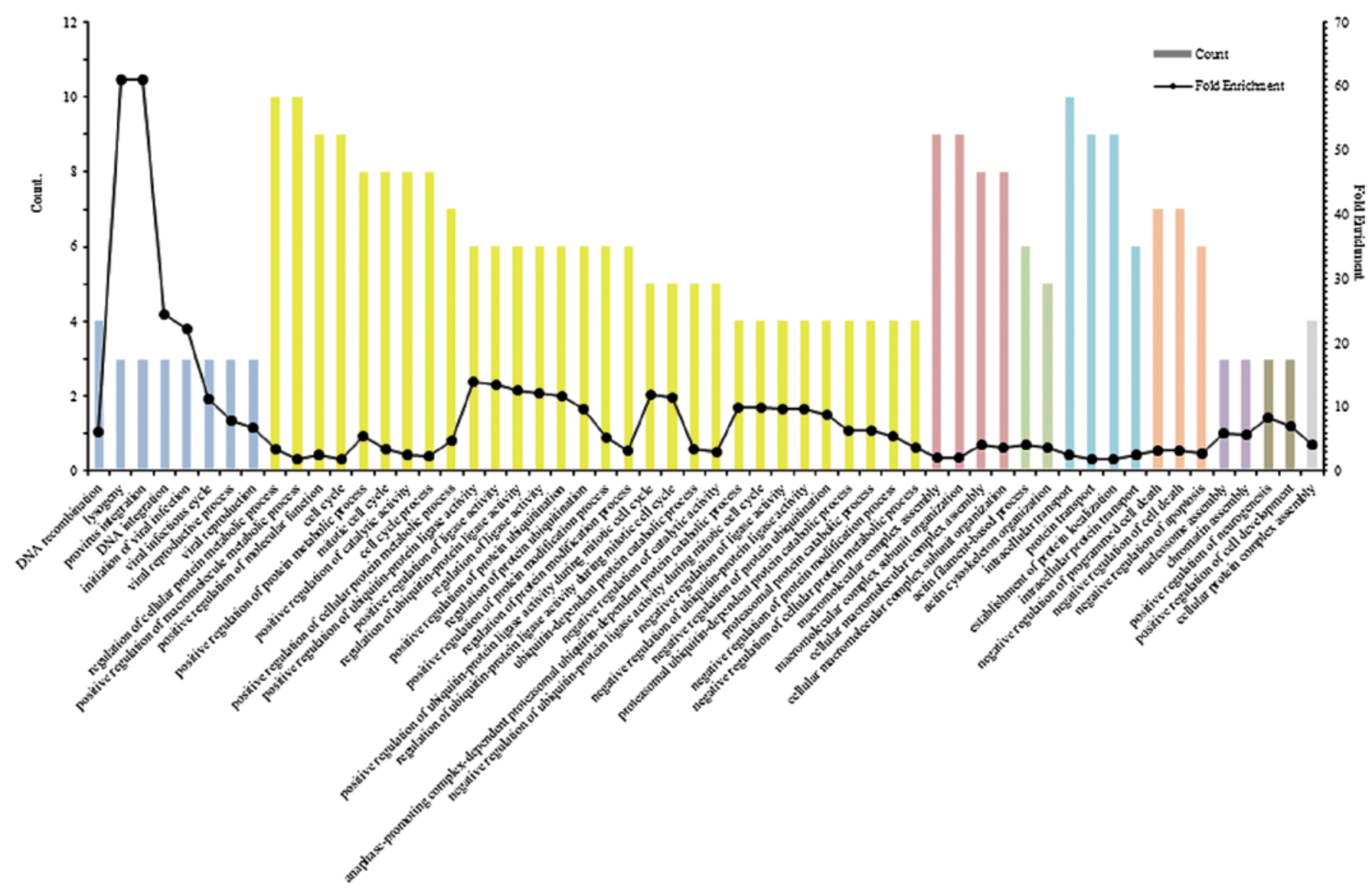

Figure 3. Functional annotation clusters ('Biological Process') in MDA-MB-231/5-FU upregulated proteins. Identification of proteins in MDA-MB-231/5-FU cells was performed by quantitative proteomics using stable isotope labeling, via iTRAQ. The most enriched clusters and characteristic examples of their Gene Ontology terms are shown, together with their enrichment scores. Bars show the count (number of included protein species). The line with the closed circles shows GO term enrichment score.

level of p53 was increased by 5-FU in MDA-MB-231, but not in its 5-FU-resistant counterpart (Fig. 2B). These results suggested that DNA damage due to 5-FU was avoided by the overexpression of $\mathrm{ABC}$ family proteins.
Quantitative differential proteomics in MDA-MB-231/5FU cells. To characterize MDA-MB-231/5-FU, we performed quantitative differential proteomic analysis of MDA-MB231/5-FU cells and the parent cells based on the iTRAQ 
Table II. Identification of upregulated proteins in MDA-MB-231/5FU cells.

\begin{tabular}{|c|c|c|c|}
\hline Accession no. & Protein name & $117 / 114$ & P-value \\
\hline gil4501881 & Actin, $\alpha$ skeletal muscle & 6.870 & \\
\hline gil62750354 & Matrin-3 isoform a & 3.242 & \\
\hline gil9257257 & WD repeat-containing protein 1 isoform 1 & 2.333 & 0.007 \\
\hline gil156523970 & $\alpha$-2-HS-glycoprotein preproprotein & 2.216 & 0.000 \\
\hline gil4506145 & Trypsin-1 preproprotein & 2.193 & 0.000 \\
\hline gil62414289 & Vimentin & 2.014 & 0.000 \\
\hline gil4503515 & Eukaryotic translation initiation factor 3 subunit $\mathrm{H}$ & 1.906 & \\
\hline gil5803013 & Endoplasmic reticulum resident protein 29 isoform 1 precursor & 1.905 & 0.037 \\
\hline gil28373194 & Proteasomal ubiquitin receptor ADRM1 precursor & 1.893 & \\
\hline gil5031635 & Cofilin-1 & 1.821 & 0.000 \\
\hline gil4507879 & Voltage-dependent anion-selective channel protein 1 & 1.781 & 0.013 \\
\hline gil50053795 & Eukaryotic translation initiation factor $4 \mathrm{~B}$ & 1.679 & 0.002 \\
\hline gil167614506 & Plastin-2 & 1.669 & 0.028 \\
\hline gil4758516 & Hepatoma-derived growth factor isoform a & 1.663 & 0.015 \\
\hline gil4758756 & Nucleosome assembly protein 1-like 1 & 1.575 & 0.000 \\
\hline gil112380628 & Lysosome-associated membrane glycoprotein 1 precursor & 1.567 & \\
\hline gil4503481 & Elongation factor $1-\gamma$ & 1.560 & 0.000 \\
\hline gil23110935 & Proteasome subunit $\alpha$ type- 1 isoform 1 & 1.493 & 0.024 \\
\hline gil25777713 & S-phase kinase-associated protein 1 isoform $b$ & 1.490 & \\
\hline gil19743823 & Integrin $\beta$ - 1 isoform $1 \mathrm{~A}$ precursor & 1.488 & 0.001 \\
\hline gil4506671 & $60 \mathrm{~S}$ acidic ribosomal protein $\mathrm{P} 2$ & 1.479 & 0.000 \\
\hline gil5032057 & Protein S100-A11 & 1.479 & 0.005 \\
\hline gil4757768 & Rho GDP-dissociation inhibitor 1 isoform a & 1.454 & 0.003 \\
\hline gil5901912 & Calmodulin & 1.448 & 0.001 \\
\hline gil386642862 & Threonine-tRNA ligase, cytoplasmic isoform 2 & 1.444 & 0.010 \\
\hline gil4758484 & Glutathione S-transferase $\omega-1$ isoform 1 & 1.441 & 0.023 \\
\hline gil4504251 & Histone H2A type 2-A & 1.429 & 0.021 \\
\hline gil6031192 & Phosphate carrier protein, mitochondrial isoform a precursor & 1.427 & 0.024 \\
\hline gil10863927 & Peptidyl-prolyl cis-trans isomerase A & 1.414 & 0.001 \\
\hline gil73486658 & Aspartate aminotransferase, mitochondrial precursor & 1.396 & 0.019 \\
\hline gil119395750 & Keratin, type II cytoskeletal 1 & 1.388 & 0.004 \\
\hline gil385298707 & Hippocalcin-like protein 1 & 1.370 & 0.005 \\
\hline gil50592994 & Thioredoxin isoform 1 & 1.356 & 0.045 \\
\hline gil4503471 & Elongation factor $1-\alpha 1$ & 1.305 & 0.002 \\
\hline gil24307939 & T-complex protein 1 subunit $\varepsilon$ & 1.297 & 0.003 \\
\hline gil4758950 & Peptidyl-prolyl cis-trans isomerase B precursor & 1.289 & 0.009 \\
\hline gil38327039 & Heat shock $70 \mathrm{kDa}$ protein 4 & 1.286 & 0.003 \\
\hline gil42544159 & Heat shock protein $105 \mathrm{kDa}$ & 1.258 & 0.008 \\
\hline gil98986464 & Transmembrane emp24 domain-containing protein 10 precursor & 1.242 & 0.002 \\
\hline gil4758012 & Clathrin heavy chain 1 & 1.221 & 0.011 \\
\hline gil5453603 & T-complex protein 1 subunit $\beta$ isoform 1 & 1.215 & 0.022 \\
\hline gil4506663 & $60 \mathrm{~S}$ ribosomal protein $\mathrm{L} 8$ & 1.206 & 0.041 \\
\hline gil5901922 & Hsp90 co-chaperone Cdc37 & 1.204 & 0.045 \\
\hline
\end{tabular}

117/114: Ratios between the two groups of 114, MDA-MB-231; and 117, MDA-MB-231/5-FU.

technique. As a result, 93 proteins with a change of expression of $\geq 1$.2-fold were considered to be upregulated, whereas 85 proteins with a change $<0.8$-fold were downregulated. Table II shows the proteins for which there was a change of expression $\geq 1$.2-fold that was significant at the level of $\mathrm{P}<0.05$. To evaluate the functional differences between parent cells and MDA-MB231/5-FU cells, we performed enrichment analysis (Fig. 3). The upregulated proteins ( $\geq 1.2$-fold) were classified into the 
GO categories of 'DNA recombination', 'cell cycle', 'complex assembly', 'cytoskeleton organization', 'transport', 'negative regulation of cell death', 'chromatin organization', and 'cell differentiation'. The enrichment scores for 'DNA recombination', 'cell cycle' and 'complex assembly' were 1.98, 1.95 and 1.81 , respectively.

\section{Discussion}

In this study, a 5-FU-resistant TNBC cell line was established from the TNBC cell line MDA-MB-231 by continuous exposure to stepwise increases in the concentration of 5-FU. The $\mathrm{IC}_{50}$ of 5-FU for the 5-FU-resistant MDA-MB-231 was significantly increased compared with that for MDA-MB-231. Moreover, MDA-MB-231/5-FU acquired cross-resistance to VNB, PTX and GEM. To the best of our knowledge, this is the first study on the establishment of a 5-FU-resistant TNBC cell line. MDA-MB-231/5-FU should be useful to study the mechanisms underlying the 5-FU resistance of TNBCs.

Recent studies have reported several determinants of 5-FU resistance mechanisms $(15,26,27)$. For instance, TS, a 5-FU-targeting enzyme; DPYD, a 5-FU-degrading enzyme; and OPRT, a 5-FUanabolic enzyme, play key roles in the 5-FU metabolism pathway. A previous study reported that the expression of DPYD and TS was enhanced in 5-FU-resistant cell lines (12). However, the expression of DPYD and TS was not enhanced in MDA-MB-231/5-FU. This suggests that the mechanisms of 5-FU resistance of MDA-MB-231/5-FU differ from those generally reported previously. Of note, MDA-MB231/5-FU showed cross-resistance to other anticancer drugs, such as PTX, VNB and GEM. Likewise, it was reported that acquisition of 5-FU resistance led to the acquisition of crossresistance to other anticancer drugs in gastric cancer cells (16). Multiple drug resistance describes a phenomenon whereby resistance to one drug is accompanied by resistance to other drugs whose structures and mechanisms of action may be completely different. Mechanisms of multiple drug resistance have been associated with increased drug efflux from cells, which is mediated by an energy-dependent mechanism (8).

The ABC family proteins, which include MDR1 and BCRP, play key roles in multiple drug resistance in breast cancer (6-8). Overexpression of MDR1 confers resistance to a variety of anticancer drugs, which are structurally and functionally unrelated, including vincristine, VNB, etoposide, PTX and many others. The expression of MDR1 and BCRP is increased in MDA-MB-231/5-FU. The overexpression of these proteins may thus be related to the partial contribution of drug efflux to multiple drug resistance in these newly established cells. To consider what kind of protein expression is enhanced other than that of $\mathrm{ABC}$ family proteins, we performed iTRAQbased quantitative proteomics on MDA-MB-231/5-FU and the parent cells. The upregulated proteins were classified into the GO categories of 'DNA recombination', 'cell cycle', 'complex assembly', 'transport' and 'negative regulation of cell death'. These results suggest that MDA-NB-231/5-FU cells were resistant to 5-FU by the enhancement of DNA recombination, regulation of the cell cycle, homologous recombination and antiapoptotic functions. These categorized proteins can be related to mechanisms of drug resistance in MDA-MB-231/5-FU. $\mathrm{S}$-phase kinase-associated protein 1 ( $\mathrm{Skp1})$, categorized as being involved in 'DNA recombination', exhibited a 1.49-fold increase in MDA-MB-231/5-FU compared with that in the parent cells. Skp1 is composed of the Skp, Cullin and F-box (SCF)-containing complex, which plays an important role in regulating the ubiquitination of specific protein substrates and regulators of cell cycle progression and development. Skp1 binds directly to F-box motifs found in F-box proteins, such as Skp2, FBW7 and $\beta$-transducin repeat-containing protein $(28,29)$. SCF protein complex regulates Akt ubiquitination, glycolysis and tumorigenesis in breast cancer (30). MDA-MB-231/5-FU may thus show enhanced ubiquitination and cell cycle progression because of the overexpression of Skp1. Likewise, peptidyl-prolyl cis-trans isomerase A, originally identified as an intracellular receptor for cyclosporine A, exhibited a 1.41-fold increase in MDA-MB-231/5-FU compared with that in the parent cells. The immunosuppressive activity of cyclosporine $\mathrm{A}$ is thought to be mediated by the engagement of calcineurin by the cyclosporin Apeptidyl-prolyl cis-trans isomerase A complex, an observation supported by the finding that peptidylprolyl cis-trans isomerase A-knockout mice are resistant to immunosuppression by cyclosporin A $(31,32)$. Peptidyl-prolyl cis-trans isomerase A was shown to be upregulated in 5-FU-treated colorectal cancer cells (33). Moreover, the overexpression of peptidyl-prolyl cistrans isomerase A induced chemoresistance to GEM (34). In this study, overexpression of $\mathrm{ABC}$ family proteins was observed in MDA-MB-231/5-FU. However, we maintain that the acquisition of multidrug resistance was not only due to the increased expression of $A B C$ family proteins. In accordance with the above findings, MDA-MB-231/5-FU should be useful to identify factors that contribute to chemoresistance in TNBCs.

Clinically, TNBC patients are treated with combination therapy of 5-FU, epirubicin and cyclophosphamide at the first-line approach. If these drugs have no effect on disease progression, PTX is applied as a second-line treatment and GEM, VNB, or other drugs as a third-line treatment. However, our 5-FU-resistant TNBC cell line acquired resistance to 5-FU, VNB, PTX and GEM. TNBCs are generally more aggressive than the standard level owing to drug resistance that developed via previous chemotherapy. This indicates that TNBC patients acquire resistance to 5-FU via the development of cross-resistance to VNB, PTX and GEM. Thus, the MDA-MB-231/5-FU established in this study should be useful for identifying new mechanisms of drug resistance and new drug targets.

\section{Acknowledgements}

The authors also wish to thank members of the Central Laboratory of Osaka City University Graduate School of Medicine, for providing technical support. This work was supported by JSPS KAKENHI Grant number 24650647 (to Katsuyuki Takahashi).

\section{References}

1. Foulkes WD, Smith IE and Reis-Filho JS: Triple-negative breast cancer. N Engl J Med 363: 1938-1948, 2010.

2. Liedtke C, Mazouni C, Hess KR, et al: Response to neoadjuvant therapy and long-term survival in patients with triple-negative breast cancer. J Clin Oncol 26: 1275-1281, 2008.

3. Thike AA, Cheok PY, Jara-Lazaro AR, et al: Triple-negative breast cancer: clinicopathological characteristics and relationship with basal-like breast cancer. Mod Pathol 23: 123-133, 2010. 
4. Dent R, Trudeau M, Pritchard KI, et al: Triple-negative breast cancer: clinical features and patterns of recurrence. Clin Cancer Res 13: 4429-4434, 2007.

5. Isakoff SJ: Triple-negative breast cancer: role of specific chemotherapy agents. Cancer J 16: 53-61, 2010.

6. Marquette $\mathrm{C}$ and Nabell L: Chemotherapy-resistant metastatic breast cancer. Curr Treat Options Oncol 13: 263-275, 2012.

7. Chen ZS and Tiwari AK: Multidrug resistance proteins (MRPs/ ABCCs) in cancer chemotherapy and genetic diseases. FEBS J 278: 3226-3245, 2011.

8. Baguley BC: Multiple drug resistance mechanisms in cancer. Mol Biotechnol 46: 308-316, 2010.

9. Abraham J, Edgerly M, Wilson R, et al: A phase I study of the P-glycoprotein antagonist tariquidar in combination with vinorelbine. Clin Cancer Res 15: 3574-3582, 2009.

10. Ruff P, Vorobiof DA, Jordaan JP, et al: A randomized, placebocontrolled, double-blind phase 2 study of docetaxel compared to docetaxel plus zosuquidar (LY335979) in women with metastatic or locally recurrent breast cancer who have received one prior chemotherapy regimen. Cancer Chemother Pharmacol 64: 763-768, 2009.

11. Zheng G, Peng F, Ding R, et al: Identification of proteins responsible for the multiple drug resistance in 5-fluorouracilinduced breast cancer cell using proteomics analysis. J Cancer Res Clin Oncol 136: 1477-1488, 2010.

12. Nakamura A, Nakajima G, Okuyama R, et al: Enhancement of 5 -fluorouracil-induced cytotoxicity by leucovorin in 5-fluorouracil-resistant gastric cancer cells with upregulated expression of thymidylate synthase. Gastric Cancer: Mar 15, 2013 (Epub ahead of print)

13. Kodera Y, Ito S, Fujiwara M, et al: Gene expression of 5-fluorouracil metabolic enzymes in primary gastric cancer: correlation with drug sensitivity against 5-fluorouracil. Cancer Lett 252 : 307-313, 2007.

14. Ichikawa W, Takahashi $\mathrm{T}$, Suto K, et al: Thymidylate synthase predictive power is overcome by irinotecan combination therapy with S-1 for gastric cancer. Br J Cancer 91: 1245-1250, 2004.

15. Longley DB, Harkin DP and Johnston PG: 5-fluorouracil: mechanisms of action and clinical strategies. Nat Rev Cancer 3: $330-338,2003$

16. Zhang X, Yashiro M, Qiu H, et al: Establishment and characterization of multidrug-resistant gastric cancer cell lines. Anticancer Res 30: 915-921, 2010.

17. Uchibori K, Kasamatsu A, Sunaga M, et al: Establishment and characterization of two 5-fluorouracil-resistant hepatocellular carcinoma cell lines. Int J Oncol 40: 1005-1010, 2012.

18. Yanagihara K, Takigahira M, Tanaka H, et al: Establishment and molecular profiling of a novel human pancreatic cancer panel for 5-FU. Cancer Sci 99: 1859-1864, 2008.

19. Kakehashi A, Ishii N, Shibata T, et al: Mitochondrial prohibitins and septin 9 are implicated in the onset of rat hepatocarcinogenesis. Toxicol Sci 119: 61-72, 2011.
20. Park JS, Young Yoon S, Kim JM, et al: Identification of novel genes associated with the response to 5-FU treatment in gastric cancer cell lines using a cDNA microarray. Cancer Lett 214: 19-33, 2004.

21. Levine AJ: p53, the cellular gatekeeper for growth and division. Cell 88: 323-331, 1997.

22. Shieh SY, Ikeda M, Taya Y, et al: DNA damage-induced phosphorylation of p53 alleviates inhibition by MDM2. Cell 91: 325-334, 1997.

23. Tibbetts RS, Brumbaugh KM, Williams JM, et al: A role for ATR in the DNA damage-induced phosphorylation of p53. Genes Dev 13: 152-157, 1999.

24. Shin JY, Kim JO, Lee SK, et al: LY294002 may overcome 5 -FU resistance via down-regulation of activated p-AKT in Epstein-Barr virus-positive gastric cancer cells. BMC Cancer 10: 425, 2010.

25. Yanamoto S, Iwamoto T, Kawasaki G, et al: Silencing of the p53R2 gene by RNA interference inhibits growth and enhances 5-fluorouracil sensitivity of oral cancer cells. Cancer Lett 223: 67-76, 2005.

26. Chu E, Drake JC, Koeller DM, et al: Induction of thymidylate synthase associated with multidrug resistance in human breast and colon cancer cell lines. Mol Pharmacol 39: 136-143, 1991.

27. Peters GJ, Backus HH, Freemantle S, et al: Induction of thymidylate synthase as a 5-fluorouracil resistance mechanism. Biochim Biophys Acta 1587: 194-205, 2002.

28. Chen J, Shen BY, Deng XX, et al: SKP1-CULLIN1-F-box (SCF)mediated DRG2 degradation facilitated chemotherapeutic drugs induced apoptosis in hepatocellular carcinoma cells. Biochem Biophys Res Commun 420: 651-655, 2012.

29. Nakayama KI and Nakayama K: Ubiquitin ligases: cell-cycle control and cancer. Nat Rev Cancer 6: 369-381, 2006.

30. Chan CH, Li CF, Yang WL, et al: The Skp2-SCF E3 ligase regulates Akt ubiquitination, glycolysis, herceptin sensitivity, and tumorigenesis. Cell 149: 1098-1111, 2012.

31. Zhu D, Cardenas ME and Heitman J: Calcineurin mutants render T lymphocytes resistant to cyclosporin A. Mol Pharmacol 50: 506-511, 1996.

32. Colgan J, Asmal M, Yu B, et al: Cyclophilin A-deficient mice are resistant to immunosuppression by cyclosporine. J Immunol 174 6030-6038, 2005.

33. Wong CS, Wong VW, Chan CM, et al: Identification of 5-fluorouracil response proteins in colorectal carcinoma cell line SW480 by two-dimensional electrophoresis and MALDI-TOF mass spectrometry. Oncol Rep 20: 89-98, 2008.

34. Kuramitsu Y, Taba K, Ryozawa S, et al: Identification of up- and down-regulated proteins in gemcitabine-resistant pancreatic cancer cells using two-dimensional gel electrophoresis and mass spectrometry. Anticancer Res 30: 3367-3372, 2010. 